$\xi=-1$

\title{
Breast Cancer Detection Using Combination of Feature Extraction Models
}

\author{
T. Suneetha Rani ${ }^{1}$, S J Soujanya ${ }^{2}$, Pole Anjaiah ${ }^{3}$ \\ ${ }^{l}$ Associate Professor, CSE Dept., QIS College of Engineering \& Technology, Ongole. Andhra Pradesh \\ ${ }^{2,3}$ Asst. Professor, CSE Dept., Institute of Aeronautical Engineering, Hyderabad. \\ *Corresponding Author Email: suneetha.tanneeru@gmail.com ${ }^{1}$,anjaiahpole@gmail.com ${ }^{3}$
}

\begin{abstract}
Recognition of either masses or tissues in a mammogram digital images is a key issue for radiologist. Present methods uses medial filter and morphological operations for detection of suspected cases in a mammogram. They use region of interest (ROI) segmentation for extraction of masses and classification of levels of severities. Classification of large number of mammogram images based on breast cancer cases takes longer computation time for performing of ROI segmentation. This is addressed by multi-ROI segmentation and it retrieves the textual properties of large mammogram images for effectively determining the breast cancer mammogram images. Experimental results shows the better performance of proposed method than existing ROI based texture feature extraction.
\end{abstract}

\section{Introduction}

Cancer is a major public health problem faced today. Detection rates of breast cancer are high in women compared to men. Breast cancer has high rate of death mortality. Appropriate deaths caused due to breast cancer increases every year. Second to lung cancer Breast cancer emerges as a rising cause of death in women .Death rates of breast cancer can be controlled through awareness of improved screening as well as early detection techniques. Breast cancer not only affects women but it also affects men. In men, it can be usually noticed through formation of hard lumps but when compared to women awareness is very little in men hence it should be checked by self-breast exam clinically and through other x-ray detection methods. Formation of lump around breast area does not always indicate the breast cancer but whenever an irregular change around the breast tissue is noticed then it should be checked up.

Breast cancer can be detected using mammograms, ultra scanning and computer based detections when viewed on mammograms by radiologist it was not easy to diagnose and breast distortion patterns that are a major signs for cancer. Major difficulty arises due to appearance of noise patterns and their uneven distribution around the breast gland. Detected results should have more great accuracy rates with reduced false positive. Mammography was commonly used method detection technique by radiologist. Mammogram images can be of two categories based on their type such as fatty, dense and glandular. Classification of cancer can be benign or malignant depending on abnormality. Training images used for this project work would be collected from the mini Mammogram image analysis society (MIAS) database. Texture analysis in image plays important role in carrying relevant information about identifying image into benign or malignant cancer. There were number of proposed texture feature extraction methods for detection of breast cancer. In this project work, four methods Were utilised local binary patterns (LBP), histogram of oriented gradients $(\mathrm{HoG})$, Gabor, Grey level co_occurence matrix (GLCM). Using advanced classifiers learning methods such as neural networks, support vector machines (SVM) accuracy rates were noted on a set of mammogram images. A support vector machine was a optimal option for learning of mammogram training data and works effectively on mammogram images classification. Implementation works of project mentioned were done using MATLAB software. Breast cancer consists of many parts like lobules, fatty, ducts and fibrous tissues. Lobules part in breast area that is also called as Glandular tissue are responsible for producing milk in women. In a breast region if there exists more number of glandular tissues without any fat in that area such a type is referred as breast density. Most of the women commonly faces breast density tissue type. These dense breast tissues along with cancerous and non-cancerous areas can be analyzed from the mammograms by concerned radiologists.

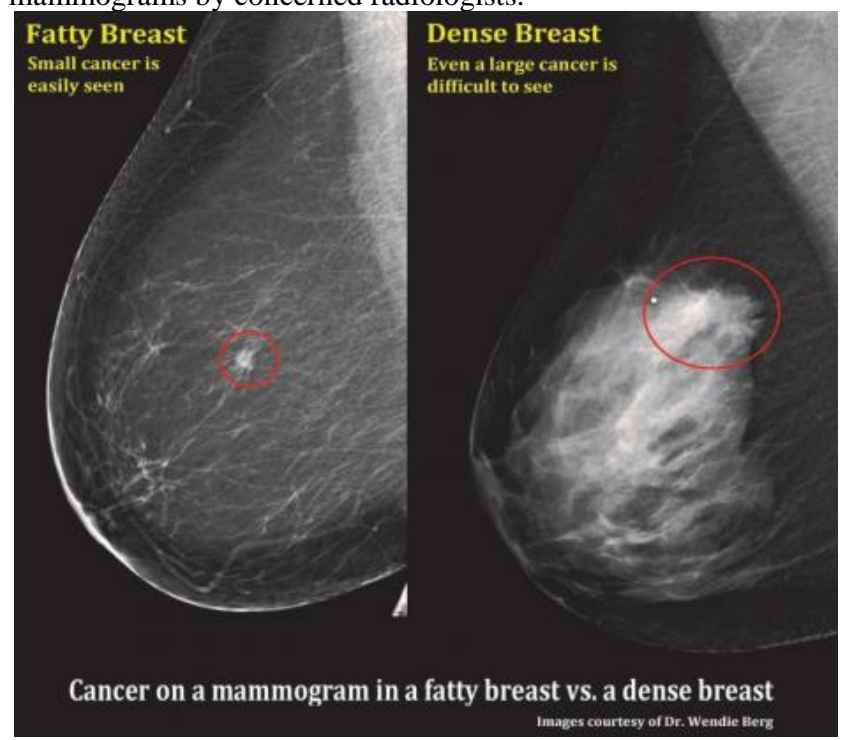

Fig. 1.1: fatty and dense mammogram images 
Figure 1.1 mentioned above shows the fatty and dense mammogram images. There are even some cases such as women with dense breast tissues type may have wrong true positive predictions, these changes can be observed more in young women compared to old aged women. In mammograms many common signs around breast tissue can be observed like lumps, ducts etc. Mammograms of screening type were used to analyze the change in Breast area such as the dense, fatty, or even lumps in some cases if they are further diagnosed based on the results from the $\mathrm{x}$ ray they can be referred as the diagnostic mammograms. In case of screening mammograms here, interesting extracted region of interest were normal and mass regions in breast tissue.

\section{Image Enhancement Techniques}

Bikeshkr.singh[7]Describes about seven enhancement methods image enhancement techniques are done generally to highlight certain features of interest in a mammogram image in this work seven enhancement methods were used within each method parameters such as contrast to noise ratios (CNR) which shows the quality improvement of images and peak signal to noise ratio (PSNR) of all the seven methods are compared if higher PSNR value then that indicates that considered method among those seven were of great quality. Among the enhancement methods one can observe that highest PSNR value can be seen in steerable filter method images in this method images are divided in to required levels by using steerable filter thus result of filtering obtained at resolution at lower level is transformed in to resolution at higher level due to this advantage additional loads can be reduced and thus improving the enhancement target of an image In case of CNR parameter one can observe that it was highest in steerable filter which indicates the quality improvement of images and least in mean filter because of inefficiency of single pixel representatives. Image enhancement method was performed after pre-processing stage. Image enhancement increases dynamic content features for image. The main aim of segmentation and classification is to extract more relevant information from extracted region of interest. In mammogram enhancement technique conventional, region based, feature based, fuzzy enhancement

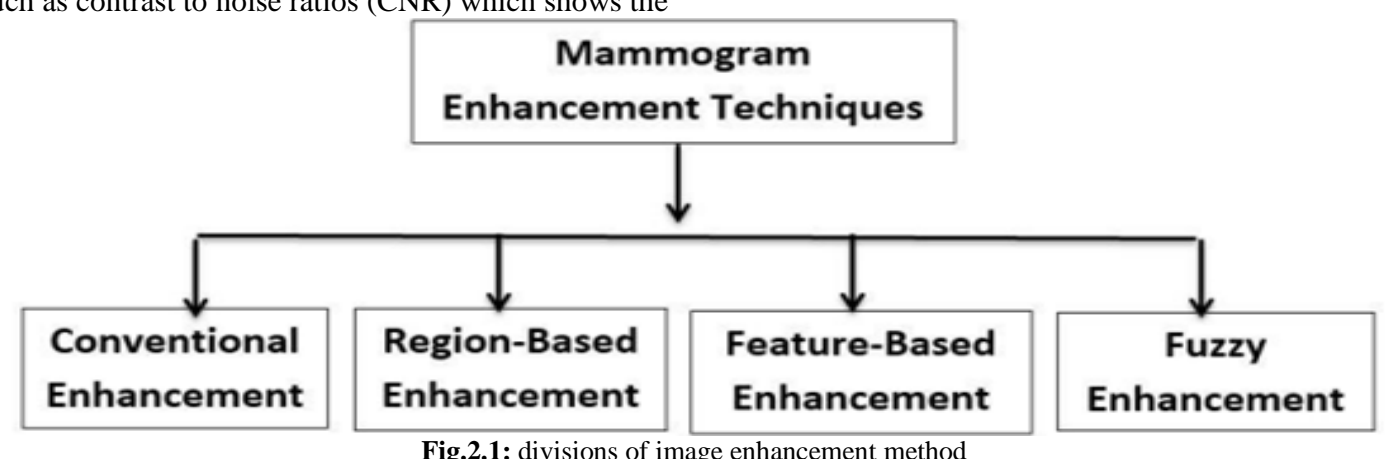

Image enhancement plays very important step in image processing applications. For any mammogram image considered initially, image enhancement would be done for pre-processed image later feature extraction method would be performed during preprocessing step image segmentation and enhancement plays important role. Image segmentation reduces the original image size. In mammograms lot of irrelevant signs were present hence segmentation was necessary step to reduce the image structure by removing unnecessary patterns. Image segmentation removes noise content for pre-processed image and improves the clarity. Image enhancement increases the dynamic content of image. There are many image enhancement methods . Figure 2.2 mentions about flow diagram taking an sample input image

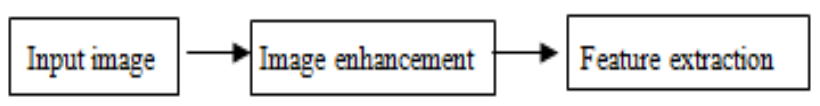

Fig.2.2: Flow diagrams for detection

\section{Segmentation}

Segmentation is a central problem and it is used to differentiate the masses or relevant objects from background. Image segmentation is used to extract Region of Interest (ROI) and its boundaries from the images. Mammogram images it is important to distinguish the abnormal region from its surroundings. The shape, margin and intensity of tissue are the important criteria's to distinguish normality and abnormality in the breast tissue. Usually a high intensity and circular based objects are more likely to be illdefined masses.

Image segmentation is a fundamental task in machine vision and occurs very frequently in many image-processing applications. Texture based segmentation provides an important data to the recognition of objects. Texture is one of the visual features playing an important role in scene analysis. Intuitively, it is related to patterned variations of intensity across an image. Texture segmentation in general is composed of two steps, namely, the extraction of texture based features and secondly the grouping of these features.

\subsection{ROI Segmentation}

Mammography is the efficient imaging modality technique that is used by radiologist for initial screening test of breast cancer. Here, it is required to find efficient segmentation technique in digital mammography. Screening is the one of standard procedure for detection of earlier stages of breast cancer in digital mammography and it can be used for detection of various abnormalities. However, it is difficult to identify the masses or tumours due to uninterested regions in mammogram images. These regions may un helps for fast processing to identify the target objects or tumours. Hence, the computer-aided detection requires the special mechanism for removing of unwanted regions during mammography segmentation. Region of interest (ROI) is the most accurate and demanding technique for segmentation of mammogram images. In helps for physicians and radiologist to find abnormality at an earliest in the absence of any symptoms.

ROI segmentation follows three steps, which are collection of mammogram images, image enhancement, and segmentation. The first step shows the collection of distinguished mammogram images from MIAS. Experimental study of previous chapters is presented well established image enhancement method, namely, IMEM for obtaining the best quality of mammogram images. The original mammograms are 1024 x 1024 pixels, and most of image 


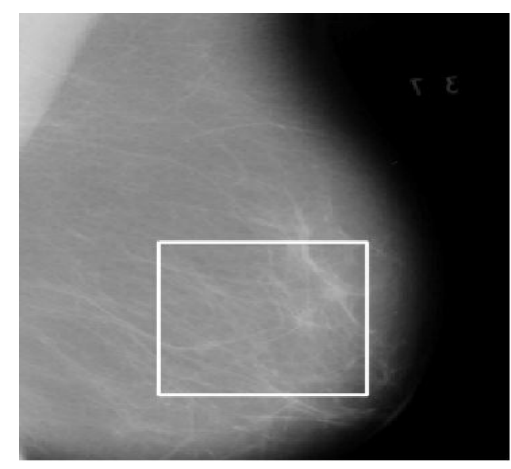

a)original image

Fig. 3.1: ROI extractions from image

Extraction of Region of interest cannot be done by physical analysis which may lead to misclassification rates. There are very works so far about automatic extraction of region of interest and segmentation. Figure mentions about extracted Region of interest from original image

\subsection{Multi-ROI Segmentation}

The basic ROI is used for finding the interested regions for single image only. For multiple images, this chapter presents another variant method, namely, multi-ROI segmentation. It performs region of interests for multiple mammogram images iteratively rather than for a single image. The basic functionality is common for both ROI and multi-ROI, however multi-ROI is an iterative version. Thus, it is flexible for selection of region of interests for large image datasets and it is faster technique. Fig. 4.1 shows the results of multi-ROI for sample mammogram images. Textual properties of segmented objects play a vital role for detection of breast cancer or classification of abnormalities of mammogram images. Feature extraction and textual properties of multi-ROI segmented images are presented in following sub-section.

\subsection{Texture Features for Mammogram Image}

\section{First-Order Statistics Based Texture Features}

The relationships with neighbourhood pixels are not considered in the first-order statistics, however these statistics computed from original values of the image and gives plenty of features based on image size I $(\mathrm{x}, \mathrm{y})$ with the size $\mathrm{M}$ x $\mathrm{N}$. These include mean, standard deviation, kurtosis, skewness, and entropy and these are described in following equations (From Eqn. 4.1 to 4.5).

$$
\operatorname{mean}(\mu)=\frac{\sum_{\mathrm{x}=1}^{\mathrm{M}} \sum_{\mathrm{y}=1}^{\mathrm{N}} \mathrm{I}(\mathrm{x}, \mathrm{y})}{\mathrm{MxN}}
$$

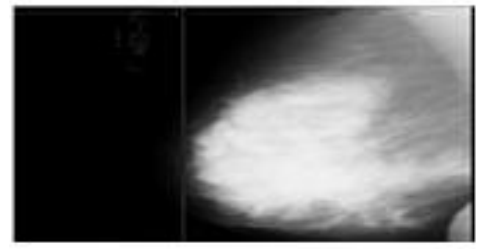

a)segmentation 1

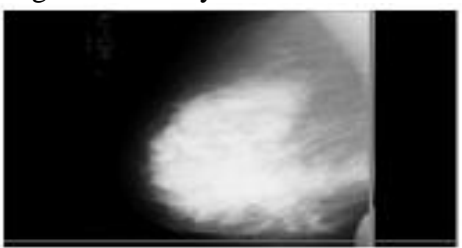

b)segmentation 2

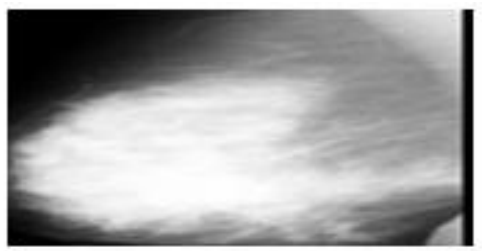

c)ROI result

Fig 3.2.1: Multi-ROI Segmentation Results for ' mdb001' image in iteration 1

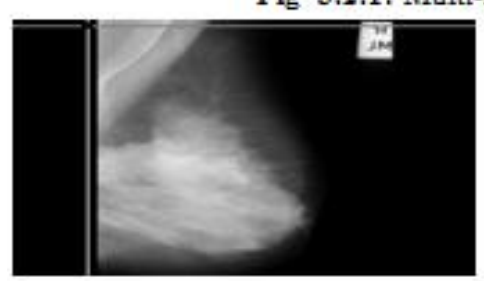

a)segmentation 1

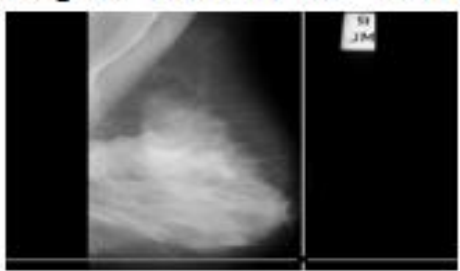

b)segmentation 2

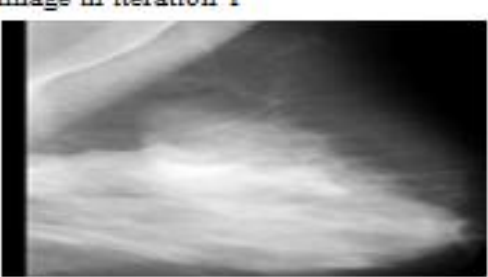

c) ROI result

Fig. 3.2.2: Multi-ROI Segmentation Results for 'mdb002' image in iteration 2

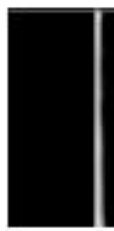

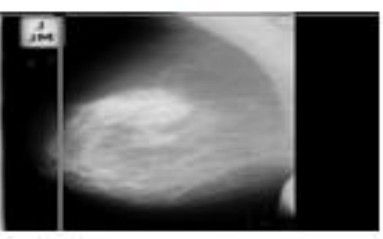

a)segmentation 1
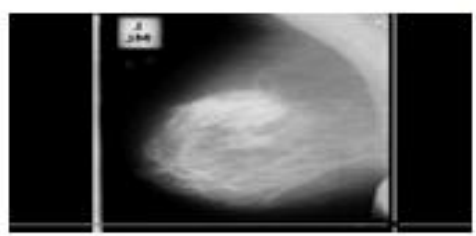

b) segmentation 2

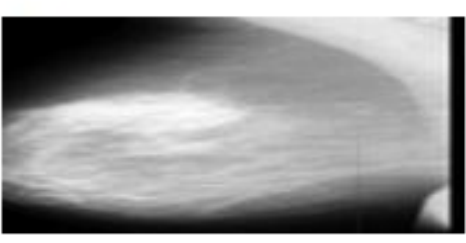

c) ROI result

Fig. 3.2.3: Multi-ROI Segmentation Results for 'mdb003' image in iteration 3

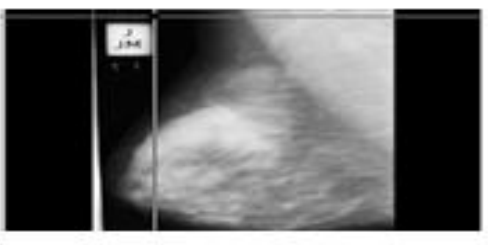

a)segmentation 1

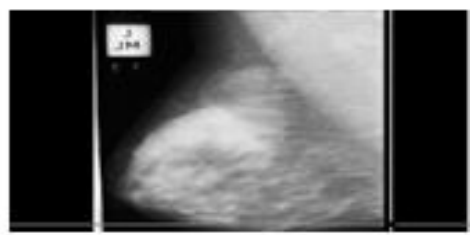

b) segmentation 2

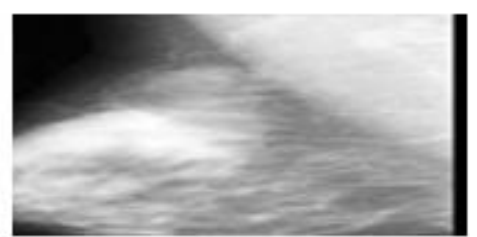

c)ROI result

Fig.3.3.4: Multi-ROI Segmentation Results for 'mdb004' image in iteration 4

Fig. 3.2: Multi-ROI Segmentation for Five Sample Images 
s tan dard_deviation $(\sigma)=\sqrt{\frac{\sum_{\mathrm{x}=1}^{\mathrm{M}} \sum_{\mathrm{y}=1}^{\mathrm{N}} \mathrm{I}(\mathrm{x}, \mathrm{y})-\mathrm{u}}{\mathrm{MxN}}}$

kurtosis $=\frac{\sum_{\mathrm{x}=1}^{\mathrm{M}} \sum_{\mathrm{y}=1}^{\mathrm{N}} \mathrm{I}(\mathrm{x}, \mathrm{y})-\mu^{4}}{\mathrm{MxNx} \sigma^{4}}$

skewness $=\frac{\sum_{\mathrm{x}=1}^{\mathrm{M}} \sum_{\mathrm{y}=1}^{\mathrm{N}} \mathrm{I}(\mathrm{x}, \mathrm{y})-\mu^{3}}{\operatorname{MxNx} \sigma^{2}}$

entropy $=\frac{1}{\mathrm{MxN}} \sum_{\mathrm{x}=1}^{\mathrm{M}} \sum_{\mathrm{y}=1}^{\mathrm{N}} \mathrm{I}(\mathrm{x}, \mathrm{y})(-\ln (\mathrm{I}(\mathrm{x}, \mathrm{y}))$

\section{Second-Order Statistics Based Texture Features}

The second-order statistics based on the relation between two neighbouring pixels in one offset, where the first pixel is called the reference and the second the neighbour pixel. These include energy, contrast, variance, correlation, homogeneity. Energy describes the regularity feature of the image. It can be defined in Eqn. (4.6).

$$
\text { energy }=\sum_{i, j=0}^{n-1} P(i, j)^{2}
$$

The variance of all pixels of mammogram image can be defined in Eqn. (4.7).

$$
\sigma^{2}=\sum_{\mathrm{i}, \mathrm{j}=0}^{\mathrm{N}-1} \mathrm{P}_{\mathrm{ij}}(\mathrm{i}-\mu)^{2}
$$

The contrast computes the variation between lowest and highest values of a neighbouring set of pixels and it shown in Eqn. (4.8)

$$
\text { contrast }=\sum_{i, j=0}^{n-1}(i-j)^{2} P(i, j)
$$

The correlation shows how a pixel is correlated to its neighbour over the whole image. It shown in Eqn. (4.9)

Correlation $=\sum_{\mathrm{i}, \mathrm{j}=0}^{\mathrm{n}-1} \frac{(\mathrm{ixj}) \mathrm{P}(\mathrm{i}, \mathrm{j})-\mu_{\mathrm{i}} \mu_{\mathrm{j}}}{\sigma_{\mathrm{i}} \sigma_{\mathrm{j}}}$

Angular second moment (ASM) is used for measuring the homogeneity of a mammogram image and Eqn. (4.10) shows the homogeneity.

$$
\text { Homogeneity }=\sum_{i=0}^{n-1} \sum_{j=0}^{n-1}\{P(i, j)\}^{2}
$$

It is experimented that to find the texture features for different visual quality of same mammogram images; since each mammogram image may appeared with different qualities using different image enhancement methods. Table 4.1 presents the value of 11 texture features; here the experiments are conducted on five benchmarked images, namely, mdb001, mbd002, mdb003, mdb004, mdb005, which are already shown in Fig. 4.1.

Table 4.1: Texture Values for Mammogram Images

\begin{tabular}{|c|c|c|c|c|c|c|}
\hline \multirow{2}{*}{$\begin{array}{c}\text { Texture } \\
\text { parameter }\end{array}$} & \multicolumn{4}{|c|}{ Name of the Image Enhancement Method } \\
\cline { 2 - 7 } & $\begin{array}{c}\text { Without Multi- } \\
\text { ROI } \begin{array}{c}\text { Pre- } \\
\text { Processed Image }\end{array}\end{array}$ & $\begin{array}{c}\text { Multi-ROI } \\
\text { Pre-processed } \\
\text { Image }\end{array}$ & $\begin{array}{c}\text { Without Multi- } \\
\text { ROI } \\
\text { Processed Image } \\
\text { Pre- }\end{array}$ & $\begin{array}{c}\text { Multi-ROI } \\
\text { Pre-processed } \\
\text { Image }\end{array}$ & $\begin{array}{c}\text { Without Multi- } \\
\text { ROI } \\
\text { Pre- } \\
\text { Processed } \\
\text { Image }\end{array}$ & $\begin{array}{c}\text { Multi-ROI } \\
\text { Pre-processed } \\
\text { Image }\end{array}$ \\
\hline Mean & 0.003491316 & 0.0038287 & 0.003491316 & 0.003368146 & 0.003491 & 0.003548 \\
\hline Standard Deviation & 0.08973598 & 0.2512469 & 0.08973598 & 0.08974516 & 0.089736 & 0.089742 \\
\hline Entropy & 2.96241 & 2.966462 & 2.96241 & 2.953132 & 2.96241 & 2.972652 \\
\hline Variance & 0.008047196 & 0.008023004 & 0.008047196 & 0.00802452 & 0.008047 & 0.008027 \\
\hline Smoothness & 0.912935 & 0.9324936 & 0.912935 & 0.9180522 & 0.912935 & 0.927643 \\
\hline Kurtosis & 12.01304 & 11.866092 & 12.01304 & 12.045874 & 12.01304 & 12.20598 \\
\hline Skewness & 1.0039384 & 1.0681014 & 1.0039384 & 1.0752846 & 1.003938 & 1.059112 \\
\hline Contrast & 0.2891548 & 0.2867634 & 0.2891548 & 0.2830924 & 0.289155 & 0.282759 \\
\hline Correlation & 0.13448966 & 0.1517824 & 0.13448966 & 0.160724 & 0.13449 & 0.142151 \\
\hline Energy & 0.7973144 & 0.7891876 & 0.7973144 & 0.7911768 & 0.797314 & 0.782127 \\
\hline Homogeneity & 0.9425092 & 0.9394728 & 0.9425092 & 0.9406992 & 0.942509 & 0.940723 \\
\hline
\end{tabular}
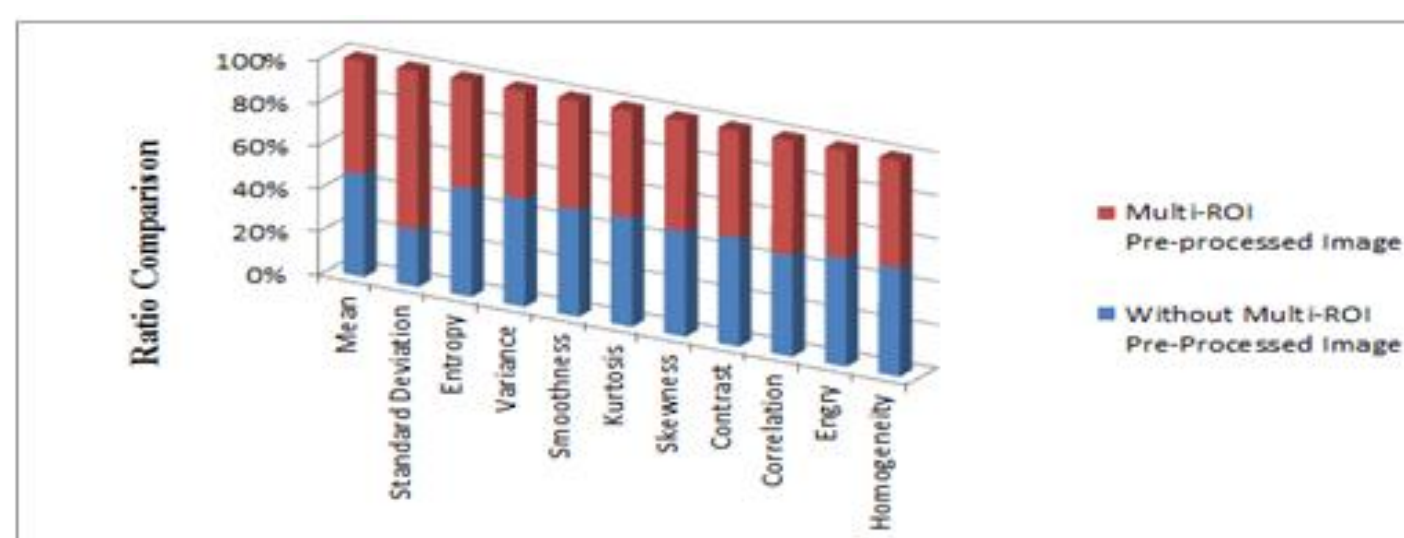

Texture Features 


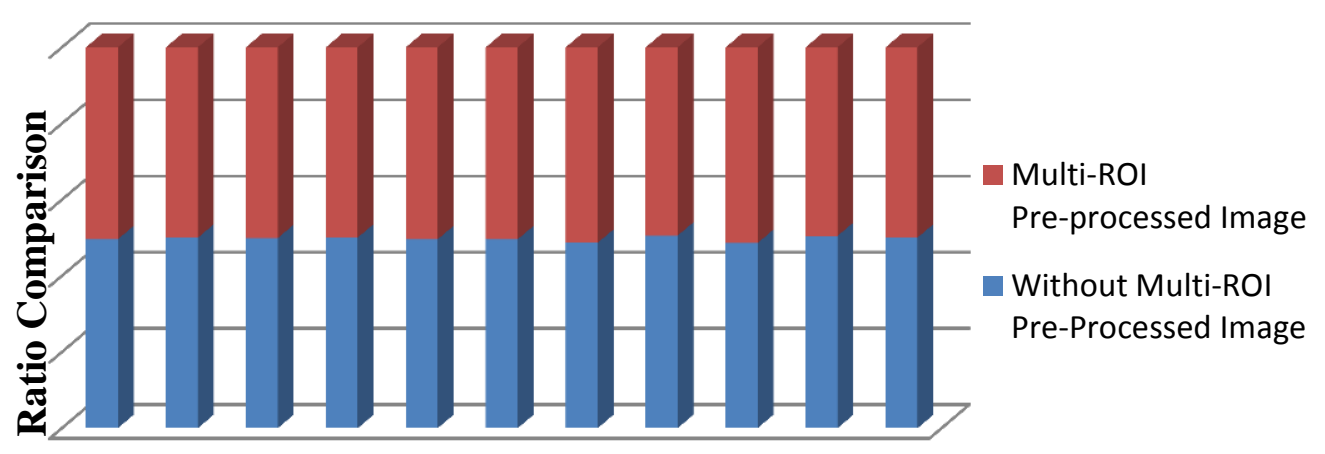

Texture Features

Texture Features Comparisons for Morphological Pre-Processed Images

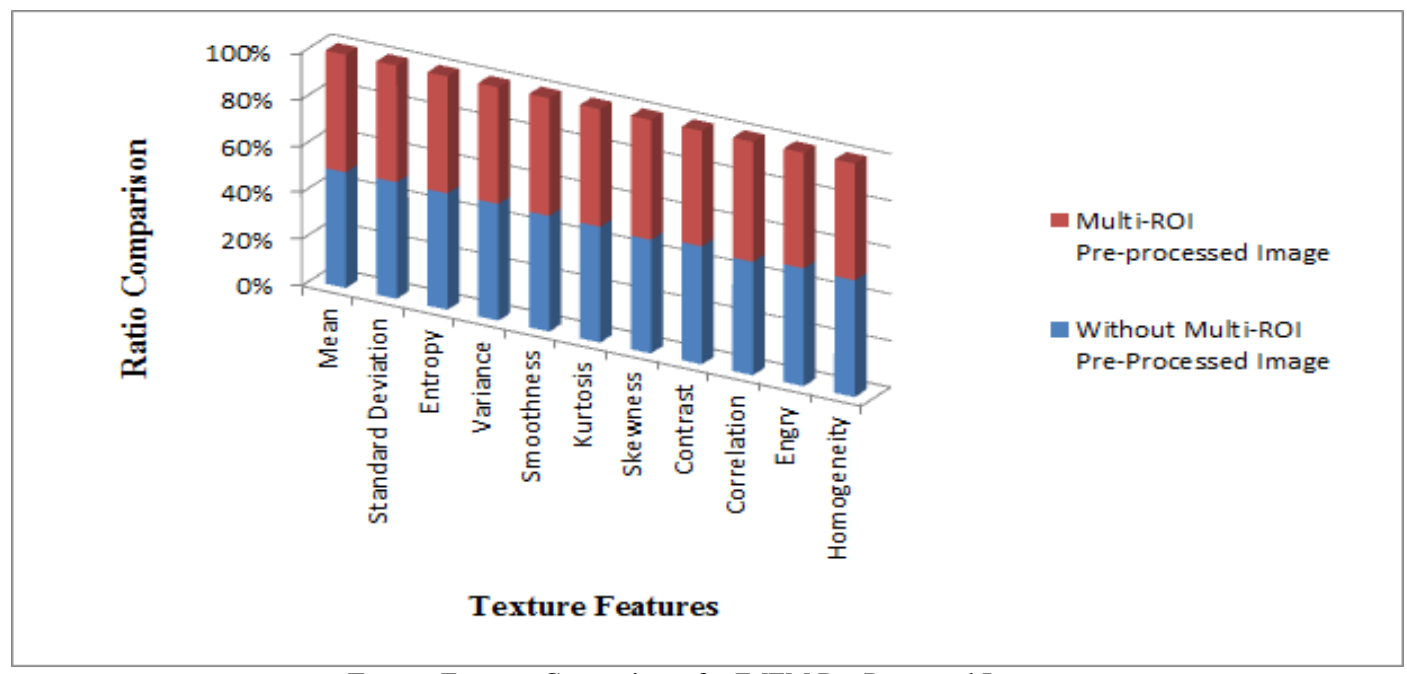

Texture Features Comparisons for IMEM Pre-Processed Images

\section{Conclusion}

Radiologists required the effective diagnosis methods for mammogram classification to the purpose of early stages of breast cancer detection that saves women life. . Automatic classification works best on a binary image that detects normal and abnormal images. Mammogram images with more number of masses are believed to having the highest statistical measure mean when compared to normal images.. Aim of computer aided diagnosis (CAD) system and mammograms was to avoid the second opinion for the detection of disease. For micro-calcifications of small size it is not easy to extract the information using morphological operations. Hence there was need for effective enhancement and segmentation steps during image pre-processing stage. Implementation work of the paper proposes the mammogram enhancement method based Region of Interest (ROI) segmentation for removing noise at a maximum extent that ultimately produces the more enhanced mammogram image.

\section{References}

[1] K. Rajendra Prasad, C. Raghavendra, K Sai Saranya, "A Review on Classification of Breast Cancer Detection using Combination of The Feature Extraction Models", International Journal of Pure and Applied Mathematics, Volume 116 No. 21 2017, 203-208.

[2] Anu Alias et.al ., "Detection of Breast Cancer Using Artificial Neural Networks", International Journal of Innovative Research in Science, Engineering and Technology Vol. 3, Issue 3, March 2014.

[3] K. Rajendra Prasad, C. Raghavendra, Effective Mammogram Classification Using Various Texture Features, Journal of
Advanced Research in Dynamical and Control Systems, Vol. 9. Sp- 12 / 2017.

[4] Dr.K.Revathyet.al., "Applying EM Algorithm for Segmentation of Textured Images", Proceedings of the World Congress on Engineering 2007 Vol I.

[5] K.Rajendra Prasad, C.Raghavendra,Padakandla Vyshnav, "Intelligent System for Visualized Data Analytics A Review", International Journal of Pure and Applied Mathematics, Volume 116 No. 21 2017, 217-224.

[6] Mar'sa V. Sainz de Cea et.al, "Estimating the Accuracy Level Among Individual DetectionClusteredMicrocalcifications", IEEE Transactions on Medical Imaging

[7] Zhili Chen et.al., "Topological Modeling and Classification of Mammographic Microcalcification Clusters", IEEE TRANSACTIONS ON BIOMEDICAL ENGINEERING, c. 62, NO. 4, APRIL 2015.

[8] Bikesh Kr. Singh et.al., "Mammographic Image Enhancement, Classification and Retrieval using Color, Statistical and Spectral" International Journal of Computer Applications, Volume 27- No.1, August 2011.

[9] S.kuma, srujanaB.jet.al., "Feature Extraction for Human Detection using HOG and CS-LBP methods" International Journal of Computer Applications,pages:11-14,2015.

[10] Snehal A. Maneet.al., "Gabor Wavelet analysis for mammogram in Breast Cancer Detection" International Journal on Recent and Innovation Trends in Computing and Communication Volume: 2 Issue: 4.

[11] C. Raghavendra, Dr. A Kumaravel, A. Anjaiah, "A New Hybrid Method for Image De-Noising In Light Of Wavelet Transform", International Journal of Pure and Applied Mathematics, Volume 116 No. 21 2017, 197-202.

[12] Varela, C., Tahoces, P. G., Méndez, A. J., Souto, M., \& Vidal, J. J. (2007). Computerized detection of breast masses in digitized mammograms. Computers in Biology and Medicine, 37(2), 214226 
[13] C. Raghavendra, A. Kumaraveland S. Sivasuramanyan, "Features Subset Selection using Improved Teaching Learning based Optimisation (ITLBO) Algorithms for Iris Recognition", Indian Journal of Science and Technology, Vol 10(34), DOI: 10.17485/ijst/2017/v10i34/118307, September 2017.

[14] Mohamed Abdel-Nasser et.al., "Improvement of Mass Detection In Breast X-Ray Images Using Texture Analysis Methods", Artificial Intelligence Research and Development, doi:10.3233/978-1-61499. 452-7-159.

[15] P. Kiran Kumar, C. Raghavendra, Dr. S. Sivasubramanyan, "Exploring Multi Scale Mathematical Morphology for Dark Image Enhancement", International Journal of Pharmacy and Technology, Dec-2016, Vol. 8, Issue No.4, 23590-23597.

[16] Zheng, Y., Breast cancer detection with Gabor features from digital mammograms, Algorithms 3.1 (2010), 44-62.

[17] Pomponiu, Victor, et al., Improving breast mass detection using histogram of oriented gradients, SPIE Medical Imaging, International Society for Optics and Photonics, 2014

[18] Ramirez R. et al., Local directional number pattern for face analysis: Face and expression recognition, , IEEE Transactions on Image Processing 22.5 (2013), 1740-1752.

[19] C. Nalini, C. Raghavendra, K. Rajendra Prasad, "Comparative Observation and Performance Analysis of Multiple Algorithms on Iris Data", International Journal of Pure and Applied Mathematics, Volume 116 No. 9 2017, 319-325.

[20] Haralick, R. M., et al., Textural features for image classification, IEEE Transactions on Systems, Man and Cybernetics 6 (1973), 610-621.

[21] Aswini Kumar Mohanty et al.," Classifying Benign and Malignant Mass using GLCM and GLRLM based Texture Features from Mammogram", International Journal of Engineering Research and Applications Vol. 1, Issue 3, pp.687-693.

[22] M.venkatramana.,et.al,review of recent texture classification:methods, IOSR journal of computer engineering22788727Volume 14, Issue 1 (Sep. - Oct. 2013), PP 54-60. 